\title{
Ein Blick auf Coachingprozesse - Die Interventionen des Coachs, dargestellt und analysiert mittels des $\mathrm{KaSyCo-C}$
}

\author{
Silvia Deplazes $^{1} \cdot$ Sandra Schwyter $^{1} \cdot$ Heidi Möller $^{2}$
}

Online publiziert: 15 . Februar 2016

(C) Die Autor(en) 2016. Dieser Artikel ist auf Springerlink.com mit Open Access verfügbar.

Zusammenfassung Obwohl in den letzten Jahren einige Forschungsarbeiten zu Coaching entstanden sind, bleibt unklar, was Coachs im Coachingprozess eigentlich tun. Mittels Videos von neun Coachingprozessen, geführt von sechs Coaching-Expert/innen, wurde induktiv ein Kategoriensystem der verwendeten Interventionen entwickelt, welches matrixartig die Interventionsformen und inhaltlichen Foki der Intervention darstellt.

Es zeigt sich, dass Coachs knapp die Hälfte der gesamten Coachingdauer zuhören und je knapp 20\% reflektieren oder Fragen stellen. Es werden hauptsächlich geschlossene, gefolgt von offenen und deutlich weniger spezifischen Frageformen wie die Zirkulären oder Hypothetischen Fragen gestellt.

Den Inhalt der Interventionen betreffend zeigt sich ein heterogenes Bild. Am häufigsten fokussieren die Interventionen das Erleben und Verhalten der Klient/innen gefolgt von dem Fokus auf das Klientensystem.

Schlüsselwörter Coachingprozess · Interventionen · $\mathrm{KaSyCo-C}$

Silvia Deplazes

deze@zhaw.ch

Sandra Schwyter

scwy@zhaw.ch

Heidi Möller

heidi.moeller@uni-kassel.de

1 Zürcher Hochschule für Angewandte Wissenschaften, Departement Angewandte Psychologie, Zürich, Schweiz

2 Universität Kassel, Kassel, Deutschland
Spotlight on the coaching processes - representing and analyzing coaches'interventions via KaSyCo-C

\begin{abstract}
Although a few studies on coaching have been carried out over the last few years, it remains unclear until today what coaches actually do in a coaching process. Based on nine video-taped coaching processes by six coaching experts, a category system of their interventions has been inductively developed. This category system illustrates, in the form of a matrix, both the types and the thematic foci of the interventions. It is shown that coaches listen to the clients nearly half of the time, and both engage in reflection and ask questions nearly $20 \%$ of the time. Predominantly, closed questions are asked, followed by open questions and significantly less often specific question types such as circular or hypothetical questions are asked. Concerning the content of the interventions, the results show a heterogeneous picture. Most frequently, the interventions focus the clients' experiencing and behavior followed by the clients' system.
\end{abstract}

Keywords Coaching process $\cdot$ Interventions $\cdot \mathrm{KaSyCo-C}$

\section{Einleitung}

Coaching ist in aller Munde. Es hat sich zu einer anerkannten Dienstleistung entwickelt. Gerade deswegen müssen Coachs in der Lage sein, zu verstehen, was einen Coachingprozess ausmacht. Denn in der heutigen Wissensgesellschaft wird Expert/innen nicht mehr unreflektiert vertraut. Es wird nachgefragt, was in einem Angebot wirklich steckt und welche Wirkungen, aber auch Nebenwirkungen, es haben kann. Immer noch verweisen viele Coachs bei Nachfragen auf ihre Erfahrung und Erfolge. Natürlich sind Erfahrungen 
wichtig, doch bleiben sie mit Referenz auf ein Individuum subjektiv (Greif 2008b), oft geprägt durch Aspekte des Selfmarketings.

Die Coaching-Ausbildungen lehren einerseits Theorien und die idealtypische Praxis, müssen aber andererseits auch die konkrete Umsetzung vermitteln. Problematisch ist hierbei, dass sowohl auf der Theorieebene als auch im Wissen um die Umsetzung in der Praxis Forschungsbedarf besteht. So wird die Entwicklung von Theorien und Analysen zum Coachingprozess sowohl für die scientific community als auch für die Praxis als besonders bedeutsam für die Weiterentwicklung von Coaching eingeschätzt (Greif 2009).

Hier setzt der vorliegende Artikel an. Anhand von Videos werden die Interventionen in neun Coachingprozessen beobachtet und analysiert.

Dabei handelt es sich um einen Teil eines Promotionsprojektes $^{1}$ (Deplazes 2015), in welchem Interventionen und Tools der Coachs, Aktivitäten der Klienten und somit letztlich ihre Interaktion analysiert wird.

\section{Interventionen im Coaching}

Der Coach stellt Fragen, hört zu, beobachtet und vieles mehr. Diese Verhaltensweisen, die wir Menschen im Alltag intuitiv verwenden, werden im Coaching zu Interventionen.

Sie werden oft beschrieben als konkrete Schritte in der Interaktion (z. B. Argyris 1970; Strikker und Flore 2010), als auf eine bestimmte Wirkung hin ausgerichtete Kommunikation (z. B. Neumann-Wirsig 2009). Demgegenüber steht die systemische Perspektive. Sie geht davon aus, dass in jedem Coaching ein soziales System gebildet wird, das operational geschlossen und autopoietisch ist (zum systemischen Interventionsverständnis vgl. z. B. Willke 1999). Klient/innen nehmen alle Kommunikationsereignisse des Coachs innerhalb dieses sozialen Systems wahr. So kann nicht zwischen einer Intervention und einem Kommunikationsereignis ohne Interventionscharakter unterschieden werden, denn jedes Kommunikationsereignis kann in den Erlebnisstrom des Klienten eingreifen. An dieser systemischen Definition von Interventionen orientiert sich die vorliegende Arbeit, denn durch die Beobachtung von Coachingprozessen kann nicht festgestellt werden, welche Interventionen des Coachs auf eine bestimmte Wirkung ausgerichtet sind oder gar beim Gegenüber eine Wirkung auslösen. So wird jede Frage, jede Anregung und auch Mimik oder Gestik als Intervention verstanden.

\footnotetext{
${ }^{1}$ Mit finanzieller Unterstützung durch die Stiftung Suzanne und Hans Biäsch zur Förderung der Angewandten Psychologie. Herzlichen Dank.
}

\section{Der Coachingprozess aus der Forschungsperspektive}

Auch wenn in den letzten Jahren einige Forschungsarbeiten zu Coaching entstanden sind, bleibt die Datenlage unsystematisch und dünn, mit Fokus auf Evaluationen und Wirksamkeit. Die Coachingforschung hinkt der Praxis hinterher und wird von dieser noch viel zu wenig wahrgenommen. Trotzdem sind einige Überblicksarbeiten zu Coachingforschung (Greif et al. 2012; Joo 2005; Künzli 2009; Möller und Kotte 2011) und auch zwei Meta-Analysen (De Meuse et al. 2009; Theeboom et al. 2014) zur Wirksamkeit von Coaching entstanden. Sie alle kommen zum Schluss, es entstehe „der Eindruck, dass Coaching wirkt" (Künzli 2009, S. 1), fügen aber an, dass die Anzahl der Studien zu gering und die Heterogenität sowohl der Qualität der Studien als auch der Ergebnisse zu groß sei, um gesicherte Aussagen machen zu können. Die einseitige Ausrichtung auf die Wirksamkeit wird kritisiert. Möller (2001) stellte bereits vor Jahren für die Supervision fest, dass kaum gefragt wird, was Supervisor/innen mit den Supervisand/innen eigentlich machen, sondern lediglich die Effekte erforscht werden. Dasselbe Bild zeigt sich heute in Bezug auf Coaching.

So wird gefordert, die Frage auszuweiten und nicht nur $\mathrm{zu}$ untersuchen, ob Coaching wirkt, sondern zu eruieren, was Coaching ausmacht und wie es wirkt (Fillery-Travis und Lane 2006; Theeboom et al. 2014).

Im Folgenden werden vorhandene Beobachtungsstudien und die vorliegende Arbeit entlang der Wissensstruktur von Coaching nach Schreyögg (2009) eingeordnet. Schreyögg (2009) umschreibt Coaching als eine Anwendung von Sozialwissenschaften und schlägt zum Sichten und Sammeln des Wissens über Coaching eine Wissensstruktur vor. Diese umfasst fünf Ebenen:

1. Mit dem ,Meta-Modell ${ }^{`}$ umschreibt Schreyögg (2009) die ,grundlegenden anthropologischen und erkenntnistheoretischen Setzungen" (S. 48).

2. Mit der ,Theorie-Ebene ${ }^{6}$ lassen sich relevante Phänomene erfassen.

3. Die ,grundlegenden methodischen Anweisungen“ umfassen die Ziele des Modells, die Rekonstruktion des Coaching-Themas, die Wirkfaktoren, den Interaktionsstil sowie die Handhabung unterschiedlicher Settings.

4. Die „methodischen Massnahmen“ sowie die „prozessualen Anweisungen zur methodischen Applizierung“ (S. 48) werden der ,Praxeologie“ zugewiesen.

5. Schließlich münden alle im ,konkreten Handeln ${ }^{6}$ des professionellen Coachs.

Vorhandene Beobachtungsstudien fokussieren auf Wirkfaktoren (Greif et al. 2012; Behrendt 2004; Greif 2009; Greif et al. 2012; Greif et al. 2010), greifen den Wirk- 
faktor Beziehung auf (Gessnitzer und Kauffeld 2014; Ianiro et al. 2013; Ianiro und Kauffeld 2011), untersuchen die Wirkung anhand des Konstrukts der Subjektiven Theorien und der Motivation (Riedel 2003) oder fokussieren die Kommunikation (Graf et al. 2010; Stein 2009; Graf 2015). Diese Arbeiten beziehen sich in der Terminologie von Schreyögg (2009) auf Wirkfaktoren oder Interaktionsstile und lassen sich in ihrer Wissensstruktur der dritten Ebene der methodischen Anweisungen zuordnen.

Die größte Ähnlichkeit zur vorliegenden Studie hat wohl die Beobachtungsstudie von (Geißler 2009), welche ein „strukturierend-klärendes Licht“ (S. 94) auf Coachingprozesse wirft.

Anhand von Tonband-Transkripten entwickelte er induktiv ein Kategoriensystem zur Analyse von Coachingprozessen. Sowohl aus der Perspektive des Coachs als auch des Klienten werden jeweils acht Dimensionen beobachtet: Beziehung zwischen Coach und Klient, Reflexion der Problematik/Herausforderung, Coachingthematik, zeitliche Positionierung, sozialer Betrachtungsstandpunkt, zeitlicher Betrachtungsstandpunkt, Realitätsstatus und wertende Rahmung. Die Kategorien lassen sich in der Wissensstruktur nach Schreyögg (2009) ebenfalls der dritten Ebene der methodischen Anweisungen zuordnen. Trotz der heterogenen Ausprägung der Coachingprozesse leitet Geißler (2013) aus den Ergebnissen der Studie konstituierende Merkmale von Coaching ab, welche unter anderem Elemente der Praxeologie beinhalten:

- Der Sprechanteil der Klientinnen ist höher als derjenige der Coachs.

- Zwanzig Prozent aller Sprechhandlungen sind Metakommunikation und dienen der Abstimmung der Coachingprozesse.

- Sprechhandlungen, die nicht metakommunikativen Charakter haben, sind vorwiegend facilitative Sprechhandlungen (min. $80 \%$ ), thematisieren den Klienten als Handlungssubjekt (min. 60\%), sind als Fragen formuliert oder spiegeln Beiträge des Klienten (zusammen min. $40 \%$ ), beinhalten wenig Selbstoffenbarungen (höchstens $10 \%$ ) und vermitteln wenig Wissen (max. $10 \%$ ).

Die vorliegende Arbeit konzentriert sich auf Interventionen und somit die vierte Ebene (Praxeologie). Dadurch sind zwar keine Aussagen zu Wirkung und Erfolg möglich, dafür kann aber der von Möller (2001) gestellten Frage nachgegangen werden, was Coaches eigentlich tun.

Die einzige Studie auf der Ebene ,Praxeologie' stammt bisher von Wechsler (2012). Auf der Grundlage von vorhandenen Wirkmodellen (Grawe et al. 1994; Greif 2008a) entwickelt sie coachingtoolspezifische Wirkfaktoren und wendet diese inhaltsanalytisch auf die in Rauen (2009; 2011) beschriebenen Tools an.

\section{Untersuchungsmethode}

\subsection{Stichprobe}

Als Einschlusskriterien für die vorliegende Stichprobe mussten die Coaching-Expert/innen mindestens fünf Jahre Coachingerfahrung haben, zum Thema publizieren, sich in der Lehre und Weiterbildung engagieren und möglichst zum Thema forschen. Die Klient/innen waren Führungspersonen, Personen in anspruchsvollen Stabsstellen oder Selbständige mit einem echten Anliegen aus ihrem Arbeitsalltag.

Die Prozesse dauerten zwischen 19 und 79 min, insgesamt annähernd sechs Stunden (5:59:04). Allen gemeinsam war, dass es sich um Coaching zu Illustrationszwecken handelte, die vor Publikum geführt wurden.

\subsection{Vorgehen}

Um die Interventionen des Coachs möglichst vollständig und verhaltensnah zu erfassen, wurde anhand von Videos induktiv mit Hilfe der Inhaltsanalyse nach Mayring (2003) das KategorienSystem zur Analyse von CoachingprozessenPerspektive Coach (KaSyCo-C) (Deplazes 2015) entwickelt. Dieses wurde in verschiedenen Reflexionsschleifen mit Coachingexperten und -forschenden diskutiert, in Beobachterschulungen vorläufig validiert und letztlich die Intercoderreliabilitäten mit vier Personen berechnet. Dabei kam ein mit diskursiven Elementen erweitertes Vorgehen zum Einsatz: Die Kodierungen wurden in Workshops abgeglichen, Abweichungen diskutiert und fehlerhafte Kodierungen im Anschluss korrigiert (vgl. Mayring 2008). Die paarweisen $\kappa$-Werte betrugen auf der Ebene Oberkategorien zwischen 0,88 und 0,90, auf der Ebene der Kategorien zwischen 0,80 und 0,86 .

Die Videos wurden computergestützt (mit dem Programm Interact) lückenlos kodiert, wobei gemäß des Event-Sampling-Verfahrens (z. B. Bortz und Döring 2006) eine kommunikative Sinneinheit eine Kodiereinheit bildet. Wiederum kam ein diskursives Vorgehen zum Einsatz, bei dem an Kodierkonferenzen Unsicherheiten in der Zuordnung und eventuelle neue Kategorien diskutiert wurden. Die Auswertung erfolgte deskriptiv wie nachfolgend in den Ergebnissen dargestellt.

\section{Ergebnisse}

\subsection{Kategoriensystem $\mathrm{KaSyCo}-\mathrm{C}$}

Die Interventionen der Coachs sind im KategorienSystem zur Analyse von Coachingprozessen - Perspektive Coach (KaSyCo-C) (Deplazes 2015) ersichtlich. Somit liefert das Analysesystem bereits zentrale Ergebnisse. 
Das Kategoriensystem unterscheidet acht Interventionsformen, die in Oberkategorien zusammengefasst sind (vgl. Abb. 1, Spalten). Die Oberkategorie Zuhören wird als rezeptiver Anteil verstanden, der Aufmerksamkeit und Interesse von Seiten des Coachs signalisiert. Reflexion umfasst (in Anlehnung an die Gesprächspsychotherapie) bewusst gestaltete Rückmeldungen. Der Coach expliziert mit eigenen Worten, was er im bisherigen Coachingprozess verstanden hat. Dabei kann er längere Gesprächsbeiträge zusammenfassen, durch seine Reflexion akzentuieren, Themen in einen neuen Zusammenhang stellen und damit eine neue Perspektive präsentieren, ohne persönlich Stellung zu beziehen oder von sich aus neue Themen einzubringen. Die Oberkategorie Frage wird in die Frageformen Zirkuläre und Hypothetische Fragen, in Skalierungsfragen sowie offene und geschlossene Fragen gegliedert. Diese verschiedenen Frageformen werden inhaltlich aufgeschlüsselt, wobei alle inhaltlichen Kategorien allen Frageformen zugeteilt werden. Handlungsanleitungen regen die Klientin zu verbalen und nonverbalen Aktivitäten an. Die Oberkategorie Erläuterung umschreibt Interventionen, die für Transparenz und Orientierung im Coachingprozess sorgen. Bezieht der Coach persönlich Stellung, wird dies der Oberkategorie Stellungnahmen zugeteilt. Unter Humor werden Parodien, Witze und Lachen subsumiert. Übrige beinhaltet verschiedene Interventionsformen, die anderweitig nicht zugeordnet werden können und in der Datenanalyse keine gewichtige Rolle einnehmen.

Zusätzlich zu den Interventionsformen beinhaltet das $\mathrm{KaSyCo}-\mathrm{C}$ (Deplazes 2015) eine Unterteilung in fünfinhaltliche Foki (vgl. Abb. 1, Zeilen). Im Fokus Sachebene/Klientensystem wird das Coachingthema kognitiv analysiert. Im Vordergrund steht die Betrachtung des Klientensystems, welches hier einerseits eine Person in ihrer Rolle, ein Team, eine Abteilung oder eine Organisation, andererseits auch abstrakte Einheiten wie die Strategie, Struktur oder eine Theorie umfassen kann. Das Erleben und Verhalten des Klienten umfasst die rezeptive Seite

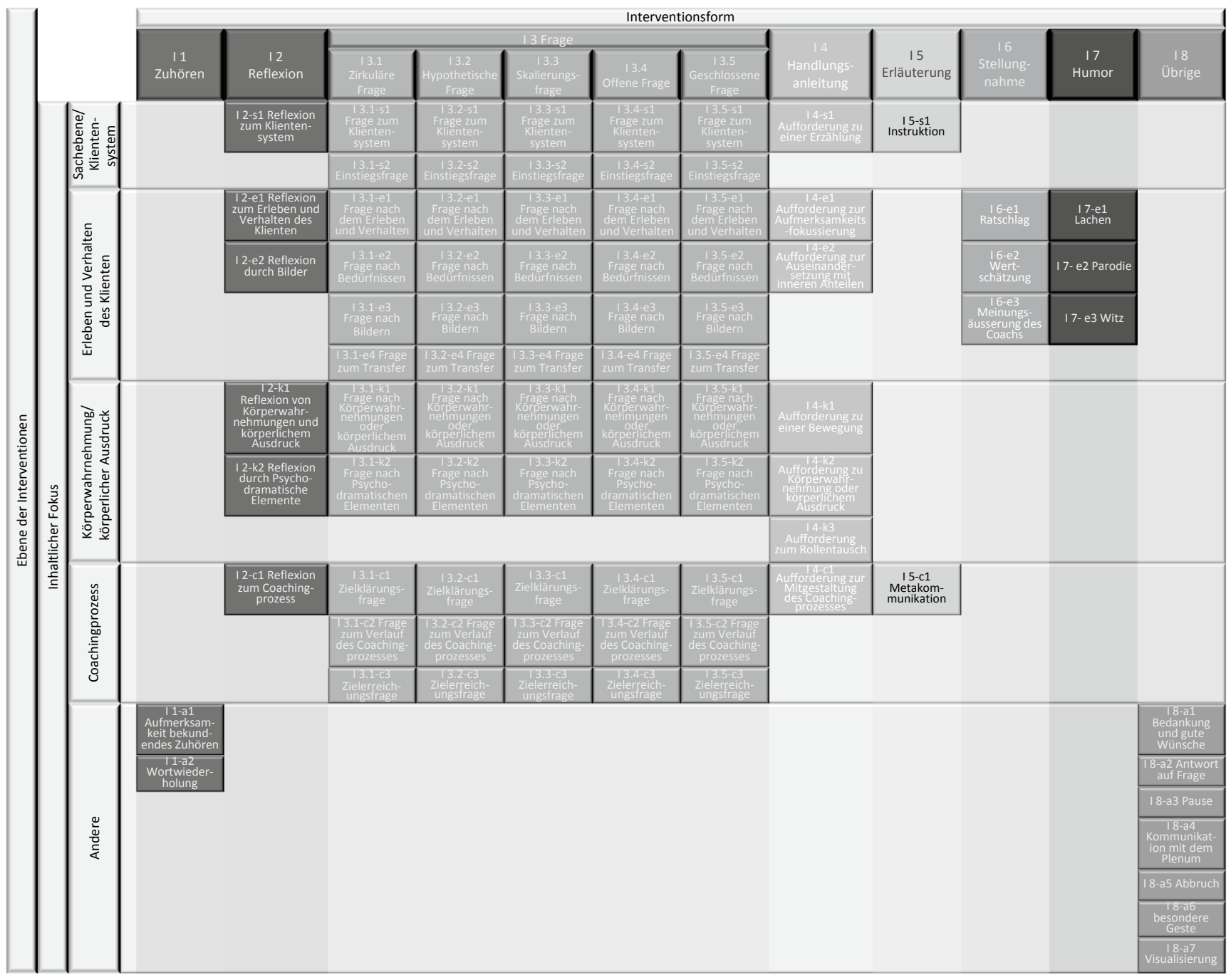

Abb. 1 Kategoriensystem zur Analyse von Coachingprozessen - Perspektive Coach (KaSyCo-C). (Deplazes 2015) 
der Interaktion zwischen der Klientin und anderen Menschen oder der Klientin und der Umwelt, sowie das Verhalten, also die beobachtbaren Aktivitäten, der Klientin. Kategorien im inhaltlichen Fokus Körperwahrnehmung/körperlicher Ausdruck zielen einerseits auf die Wahrnehmungen des Körpers, andererseits auf körperliche Darstellungen und Bewegungen ab. Der Fokus Coachingprozess liegt bei prozessstrukturierenden und Transparenz schaffenden Anteilen. Unter Andere finden sich inhaltlich nicht zu bestimmende Interventionen.

\section{Häufigkeiten und Dauer der verwendeten Interventionsformen}

Die annähernd sechs Stunden Videomaterial umfassten 3615 Kodierungen. Von den insgesamt 84 möglichen Interventionen (Kategorien) wurden innerhalb dieser Stichprobe 60 kodiert. Die 24 nicht verwendeten Interventionen (Kategorien) sind durch die Kombination der Frageformen mit allen Frageinhalten entstanden.

Alle Resultate wurden aus den Perspektiven Häufigkeit $(3615=100 \%)$ und Dauer $(21544 \mathrm{~s}=100 \%)$ betrachtet, um einer Verzerrung durch die unterschiedlich langen Kodiereinheiten vorzubeugen. Im Folgenden werden nur die wichtigsten Ergebnisse beschrieben, für weitere Informationen sei auf die Grafiken verwiesen. Abbildung 2 weist die Häufigkeiten, Abbildung 3 die Dauer der Interventionsformen aus. Sowohl bei der Häufigkeit $(31,6 \%)$ als auch bei der Dauer (45,9\%) entfiel der größte Anteil auf die Oberkategorie Zuhören. Die Oberkategorie Frage war mit 19,8\% im Vergleich zu Reflexion (18,1\%) häufiger, jedoch kürzer hinsichtlich der Dauer (Frage 14,4\%, Reflexion 17,2\%). Deutlich geringere Ausprägungen zeigten die übrigen Oberkategorien.

Bei der detaillierteren Betrachtung der Interventionen wurde ersichtlich, dass auf die Frageformen Zirkuläre resp. Hypothetische Fragen und Skalierungsfrage insgesamt nur

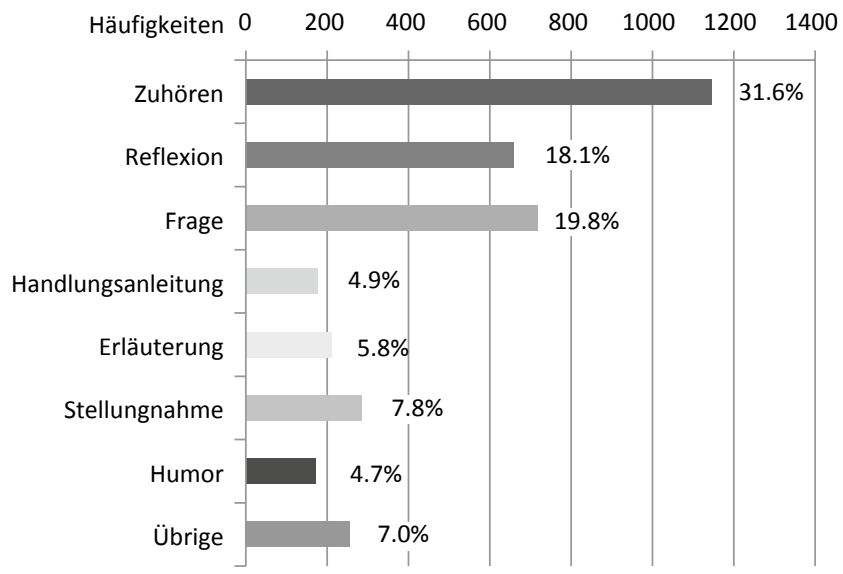

Abb. 2 Häufigkeiten und Prozent der Interventionen (3615=100\%)

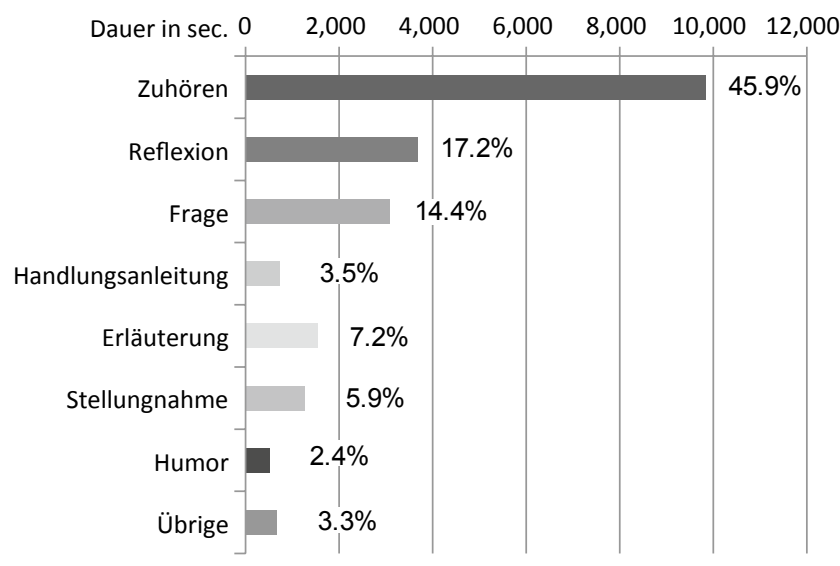

Abb. 3 Dauer (in Sekunden) und Prozent der Interventionen $(21544 \mathrm{~s}=100 \%)$

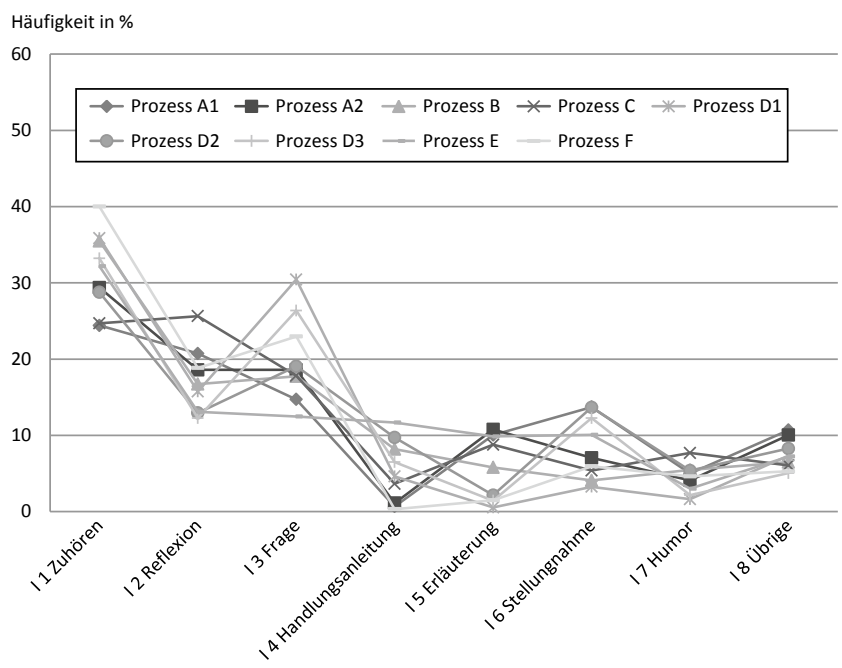

Abb. 4 Vergleich der Prozesse bezüglich der Häufigkeit der Interventionen in Prozent $(3615=100 \%)$. Die Buchstaben A bis F bezeichnen die Coaching-Experten.

2,3\% der Häufigkeit und 1,9\% der Dauer entfielen. Die Zirkuläre Frage wurde mit 60 Kodierungen häufiger als die Hypothetische Frage (21 Kodierungen) und die Skalierungsfrage (3 Kodierungen) angewendet. Die 60 Zirkulären Fragen sind teilweise darauf zurückzuführen, dass diese neben der klassischen Definition auch kodiert wurden, wenn der Klient in einer Rolle (z. B. im Rahmen der Stuhlmethode) angesprochen wurde. Die geschlossene Frage war mit 10,4\% der Häufigkeit und 7,2\% der Dauer die am meisten eingesetzte Frageform. Unter diese Kategorie fielen auch alle Aussagen, die mit einem fragenden ,oder?' resp. ,ja?' abgeschlossen wurden. Die Oberkategorie Stellungnahmen beinhaltete neben (verbalisierter) Wertschätzung auch Ratschläge (Häufigkeit 2,0\%, Dauer $3,2 \%$ ) und Meinungsäußerungen des Coachs (Häufigkeit 2,0\%, Dauer 1,4\%). Der größte Anteil von Erläuterung fiel auf die Metakommunikation (Häufigkeit 4,5\%, Dauer 4,8\%).

In Abb. 4 wird die Heterogenität der Stichprobe im Hinblick auf die Häufigkeit der Oberkategorien deutlich. 
Zuhören bewegte sich je nach Prozess zwischen knapp 25 und $40 \%$. Bei der Reflexion lag die Häufigkeit zwischen $13 \%$ und fast $26 \%$. In Prozess $\mathrm{C}$ war die Reflexion als Intervention häufiger als Zuhören. Der Anteil der Fragen lag zwischen $12,5 \%$ und über $30 \%$. Handlungsanleitungen machten 0,5 bis $12 \%$ der Interventionen aus. Große Unterschiede waren auch in der Oberkategorie Erläuterung zu finden; die Werte variierten zwischen 0,5 und $10 \%$, im Falle der Stellungnahmen zwischen 3 und 10\%.

Abbildung 5 zeigt für alle Prozesse ein grundsätzlich homogeneres Bild hinsichtlich der Dauer der Interventionen auf. Die Oberkategorie Zuhören zeigte bei allen Prozessen die höchste Ausprägung. Sie bewegte sich zwischen 39\% und über $52 \%$ der gesamten Dauer. Die Reflexion lag zwischen knapp 12 und 26\%, wobei sich die Prozesse A1 mit 19,5\% und C mit 26\% abhoben. Der Anteil Fragen bewegte sich zwischen $10 \%$ und über $21 \%$. Bei den Handlungsanleitungen lagen drei Prozesse bei unter 0,5\% und zwei Prozesse bei $10 \%$ bis fast $12 \%$ der Dauer. Vier Prozesse wiesen bei der Erläuterung zwischen 0,3 und $1,7 \%$ auf, die anderen fünf Prozesse lagen bei $7 \%$ bis über 14\%. Der Anteil der Stellungnahmen lag zwischen 3,5 und $11 \%$ der gesamten Dauer.

In Abb. 4 und Abb. 5 wird ersichtlich, dass alle Oberkategorien in allen Prozessen vorkommen. Auf der Ebene der Frageformen zeigte sich jedoch, dass nur offene und geschlossene Fragen in allen Prozessen Verwendung fanden. Zirkuläre und Hypothetische Fragen wurden in je vier der neun Prozesse verwendet, wobei nur in einem Prozess beide Fragearten angewendet wurden. Die Skalierungsfrage wurde nur in einem Prozess genutzt.

Die Heterogenität der Prozesse zeigt sich auch darin, dass lediglich zehn der insgesamt 84 Interventionen in

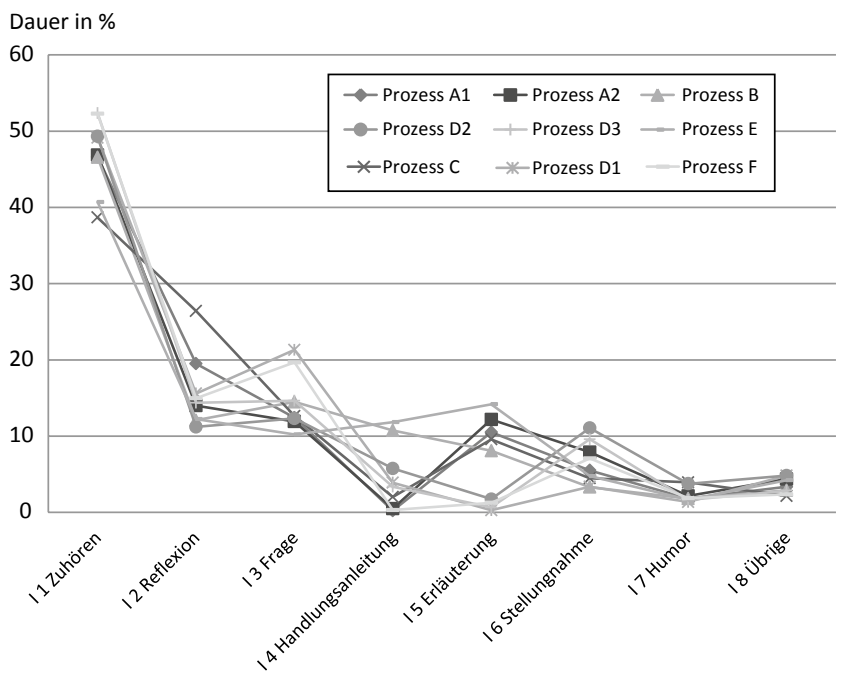

Abb. 5 Vergleich der Prozesse bezüglich der Dauer der Interventionen in Prozent $(21544 \mathrm{~s}=100 \%)$. Die Buchstaben A bis F bezeichnen die Coaching-Experten. (Erforderliche Parameter fehlen oder sind falsch) allen Prozessen vorkamen: Aufmerksamkeit bekundendes Zuhören, Paraphrase, Reflexion zum Klientensystem, Reflexion zum Erleben und Verhalten des Klienten, offene Frage nach dem Erleben und Verhalten, geschlossene Frage zum Verlauf des Coachingprozesses, Ratschlag, Wertschätzung, Lachen und Parodie.

\section{Inhaltliche Foki der verwendeten Interventionen}

Zusätzlich zu den Oberkategorien strukturiert $\mathrm{KaSyCo}-\mathrm{C}$ (Deplazes 2015) die Interventionen in inhaltliche Foki. Abbildung 6 und Abb. 7 zeigen auf, dass sich bei den inhaltlichen Foki hinsichtlich Häufigkeit und Dauer sehr ähnliche Profile ergaben. Am meisten wurde der Fokus Erleben und Verhalten des Klienten beobachtet (Häufigkeit 32,1\%, Dauer 27,8\%). Die Sachebene/das Klientensystem wurde mit 15,6\% nur halb so häufig thematisiert (Dauer 11,9\%). Der Coachingprozess lag mit 7,6\% der Häufigkeit und 7,5\% der Dauer noch knapp vor dem Fokus Körperwahrnehmung/körperlicher Ausdruck (Häufigkeit 5,7\%, Dauer 3,4\%). In Abweichung zum Kategoriensystem wird der inhaltliche Fokus Andere ohne die Oberkategorie Zuhören dargestellt. Das Zuhören wird aufgrund der Häufigkeit $(31.6 \%)$ resp. Dauer (45.9\%) separat dargestellt.

Bei den inhaltlichen Foki zeigte sich die große Heterogenität der einzelnen Coachingprozesse erneut. Diese war bei der Häufigkeit (Abb. 8) und der Dauer (Abb. 9) der Inhalte ähnlich ausgeprägt. Die Werte von Sachebene/ Klientensystem variierten bei der Häufigkeit zwischen 3,5 und $36 \%$, bei der Dauer zwischen 3 und $27 \%$. Die Anteile von Erleben und Verhalten des Klienten bewegten sich zwischen knapp 17 und 47\% (Häufigkeit) resp. zwischen 16 und $38 \%$ (Dauer).

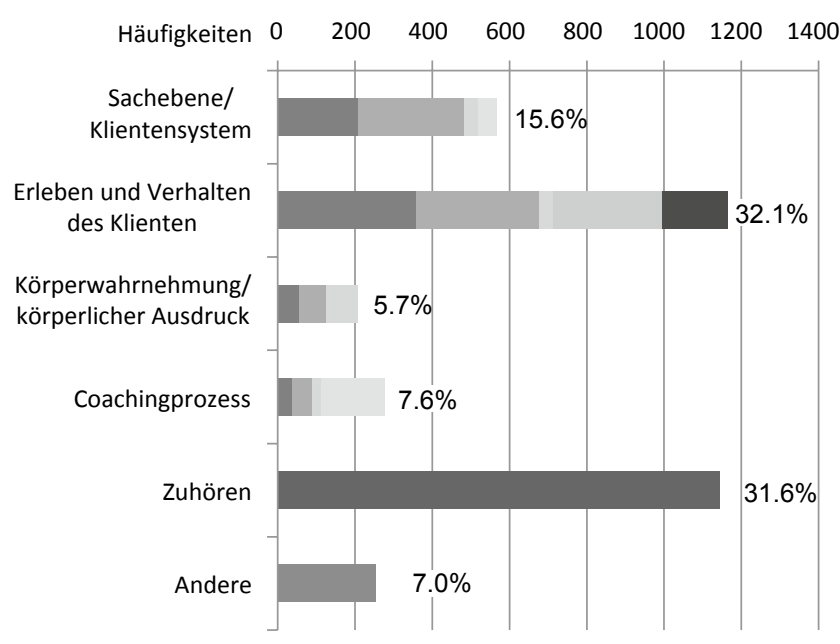

Abb. 6 Häufigkeit und Prozent der inhaltlichen Foki $(3615=100 \%)$ 


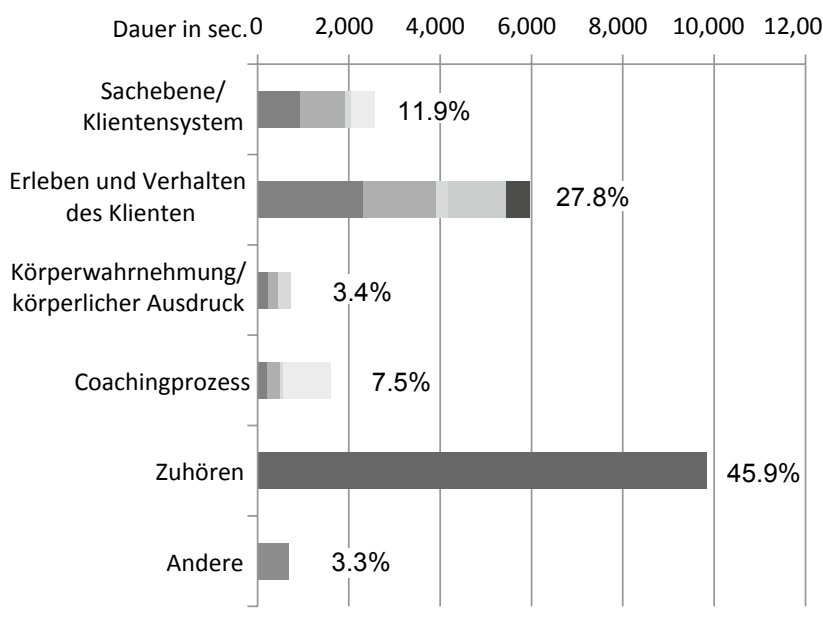

Abb. 7 Dauer (in Sekunden) und Prozent der inhaltlichen Foki $(21544 \mathrm{~s}=100 \%)$

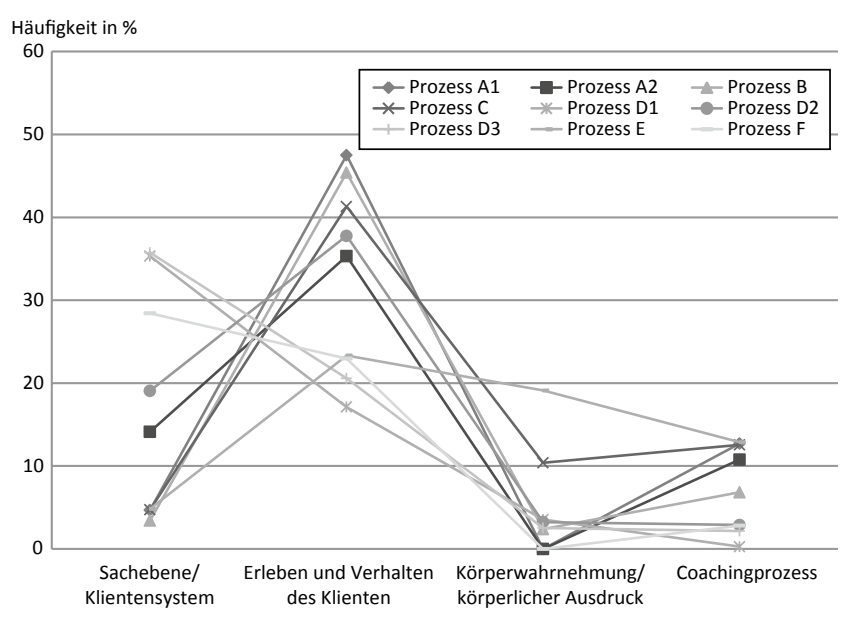

Abb. 8 Vergleich der Prozesse bezüglich der Häufigkeit der inhaltlichen Foki in Prozent $(3615=100 \%)$.

Alle Prozesse außer Prozess F wiesen große Unterschiede in der Häufigkeit der beiden Foki Sachebene/Klientensystem und Erleben und Verhalten des Klienten auf. Dabei fokussierten zwei Prozesse die Sachebene/Klientensystem und zeigten tiefere Werte im Erleben und Verhalten des Klienten, während dies bei den restlichen sechs Prozessen genau umgekehrt war.

Interventionen mit dem Fokus Körperwahrnehmung/ körperlicher Ausdruck kamen in drei Prozessen nicht und in vier Prozessen zwischen 2 und 3,5\% (Häufigkeit) resp. zwischen 0,8 und 2,5\% (Dauer) vor. In Prozess $\mathrm{C}$ lag der Fokus in $10 \%$ der Häufigkeit und $6,5 \%$ der Dauer auf Körperwahrnehmung/körperlicher Ausdruck, in Prozess E in $19 \%$ der Häufigkeit, resp. $16 \%$ der Dauer. Der Anteil mit Fokus auf den Coachingprozess variierte stark zwischen $0,3 \%$ und annähernd $13 \%$ (Häufigkeit) resp. zwischen $0,2 \%$ und über $10 \%$ (Dauer).

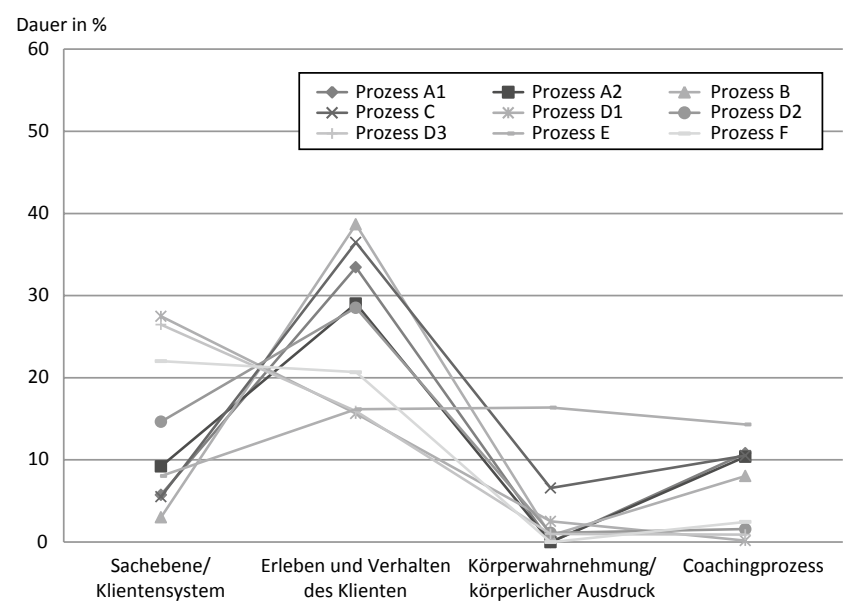

Abb. 9 Vergleich der Prozesse bezüglich der Dauer der inhaltlichen Foki in Prozent $(21544 \mathrm{~s}=100 \%)$.

\section{Diskussion}

Die Analyse hat gezeigt, welche Interventionen - im systemischen Sinne verstanden als Gesprächseinheit des Coachs - in Coachingprozessen eingesetzte werden. Wie der Blick auf den State of the Art der Coachingforschung mit Fokus auf Beobachtungsstudien offenbarte, sind nur wenige Studien zu finden, welche anhand von Videos oder Tonbändern den Prozess direkt beobachten und keine, die sich auf diese Weise vertieft mit der Praxeologie auseinandersetzen. Die vorliegende Analyse der Interventionen im Coaching ist daher eine wichtige Ergänzung zu bestehenden Studien. Das induktiv entwickelte Kategoriensystem KaSyCo-C (Deplazes 2015) stellt den Kern der Analyse dar.

\subsection{Interpretation der Ergebnisse}

Auf den ersten Blick entsprechen sowohl das Kategoriensystem als auch die ersten Ergebnisse zu Häufigkeiten und Dauer der Interventionen im Coachingprozess den Erwartungen. Die Coachs hörten am meisten zu, gefolgt von Fragen und Reflexionen. Weniger häufig waren Erläuterungen und Stellungnahmen. Ein Blick auf den Inhalt der Interventionen zeigt, dass das Erleben und Verhalten der Klienten am häufigsten thematisiert wurde, gefolgt von der Sachebene/dem Klientensystem. Deutlich weniger wurde der Coachingprozess besprochen und die Körperwahrnehmung diskutiert, resp. mit körperlichem Ausdruck gearbeitet.

$\mathrm{Zu}$ der in letzter Zeit immer wieder diskutierte Frage, ob im Coaching auch Ratschläge gegeben werden dürfen (Böning 2014), oder neben der Prozessberatung auch Expertenberatung (Schreyögg 2010; Seiger und Künzli 2013) erfolgen soll, kann durch die Daten beigetragen werden. In allen neun Prozessen wurden Ratschläge 
gegeben. Dieses Ergebnis ist umso eindrücklicher, als nur 10 der insgesamt 84 Kategorien in allen Prozessen vorkommen. Es zeigt, dass der in der Literatur oft dargestellten reinen Prozessberatung nicht gefolgt wird. Weiter nahm der Coach nur in einem Prozess nicht persönlich Stellung und brachte seine eigene Meinung nicht ein. Hier scheint die Praxis der theoretischen Auseinandersetzung zuvor zu kommen, indem bereits praktiziert wird, was in der Literatur erst langsam aufgenommen wird.

Wie auch in anderen Untersuchungen (Geißler 2009) unterscheiden sich die Prozesse stark. Dies zeigt sich einerseits, dass lediglich zehn der insgesamt 84 Interventionen in allen Prozessen vorkamen. Andererseits zeigt es sich mit Blick auf die inhaltlichen Foki der einzelnen Prozesse. In zwei Prozessen bezieht sich der Inhalt hauptsächlich auf die Sachebene/das Klientensystem, in sechs Prozessen auf das Erleben und Verhalten der Klientin und nur ein Prozess zeigt ein ausgewogenes Verhältnis dieser beiden inhaltlichen Foki. Es scheint, dass sich Coachs auf einen inhaltlichen Fokus konzentrieren. Es bleibt allerdings offen, ob diese inhaltliche Fokussierung durch das Coachinganliegen, also hauptsächlich vom Klienten beeinflusst wird, oder ob der Coach diese inhaltliche Fokussierung, z. B. durch seinen theoretischen Hintergrund prägt. Es wäre denkbar, dass z. B. Coachs mit einem psychologischen Hintergrund andere Foki setzen als Coachs mit einem betriebswirtschaftlichen Background.

Die großen Unterschiede bezüglich der Verwendung von Interventionen mit dem inhaltlichen Fokus Körperwahrnehmung/körperlichem Ausdruck (0 bis 19\%) lassen sich vermutlich eher durch die bevorzugte Arbeitsweise des Coachs erklären. Es ist anzunehmen, dass Coachs, die z. B. eine Zusatzausbildung im Zürcher Ressourcenmodell und dadurch Zugang zum Thema Embodiment (Storch und Krause 2002) mitbringen, dieses erlebniszentrierte Vorgehen eher einsetzen, während andere Coachs keinen Zugang zu solchen Vorgehensweisen haben. Eine deutliche Ausprägung diesbezüglich zeigt sich z. B. in einem Psychodrama-Coaching. Hier erfolgt explizit keine Wahl der Methode durch das Coachinganliegen. Vielmehr ist der gesamte Prozess geprägt von dieser methodischkonzeptionellen Orientierung des Coachs. Offen bleibt die Frage, ob diese Coachings als typisch für die Protagonist/ innen gelten können oder aber im Sinne einer didaktischen Funktion in der Weiterbildung und/oder auf Kongressen das Verfahren in den Vordergrund gestellt wurde.

Überraschende Ergebnisse finden sich bei einem genaueren Blick aufdie Kategorien: Fragen - oft systemische Fragen - nehmen sowohl in der Literatur (z. B. Backhausen und Thommen 2006; Radatz 2003) als auch in der Weiterbildung einen zentralen Stellenwert ein. Vor diesem Hintergrund überrascht es sehr, dass nur in einem einzigen Coachingprozess Skalierungsfragen (3 Kodierungen) vor- kamen. Hypothetische Fragen wurden nur 22 mal in vier der neun Prozesse gestellt. Die 60 Zirkulären Fragen, die ebenfalls in vier Prozessen gestellt wurden, machten nur gut acht Prozent der Fragen aus. Die größte Überraschung zeigt sich aber bei geschlossenen Fragen. Sie werden als eher ungeeignet für Coaching eingestuft (z. B. Schreyögg 2012). Mit 378 Kodierungen machen sie aber im vorliegenden Material gut die Hälfte aller Fragen aus. Es ist allerdings unbekannt, welche Wirkungen diese geschlossenen Fragen im Rahmen eines Coachings zeigen, und ob sie damit für Coachings wirklich so ungeeignet sind. Ob sich beispielsweise die Antwort der Klientinnen bezogen auf offene oder geschlossene Fragen unterscheidet, wird sich bei der Musteranalyse im weiteren Verlauf des diesem Artikel zu Grunde liegenden Forschungsprojekts zeigen.

Eine solche Abweichung von der Theorie muss also nicht negativ eingestuft werden. Die Coachs der Stichprobe sind seit vielen Jahren als Coachs aktiv und etabliert, ihre Arbeitsweise scheint erfolgreich zu sein. Nichtsdestotrotz sollte ein Diskurs darüber erfolgen, dass Theorie und Praxis offensichtlich deutlich von einander abweichen. Neben den Frageformen betrifft dies insbesondere die Ratschläge und Meinungsäußerungen des Coachs und damit die direktiveren Interventionen im Coaching. Hier sollte im Rahmen weiterer Analysen der Frage nachgegangen werden, ob Beeinflussung (doch) zum Coaching gehört und wenn ja, wie diese gestaltet werden sollte. Wäre es diesbezüglich z. B. sinnvoll mit Wahlmöglichkeiten zu arbeiten?

\subsection{Konstitutive Merkmale von Coaching}

Lohnend scheint der Vergleich der vorliegenden Ergebnisse mit den von Geißler (2013) definierten konstitutiven Merkmalen von Coaching. Dabei zeigen sich neben einigen Übereinstimmungen auch Unterschiede. Gleichzeitig ist dieser Vergleich jedoch mit Vorsicht zu betrachten, da die Kategorien nach Geißler (2013) und diejenigen des KaSyCo-K (Deplazes 2015) zwar Überschneidungen zeigen, aber nicht deckungsgleich sind. Außerdem bezieht sich Geißler ausschließlich auf Sprechhandlungen. Daher wurde die Oberkategorie Zuhören für die folgenden Vergleiche ausgeschlossen; die Prozentzahlen unterscheiden sich folglich von den im Ergebnisteil dargestellten Werten. Trotz aller Einschränkungen scheint der Vergleich mit diesen konstitutiven Merkmalen lohnend, sind es doch die einzigen Aussagen, welche aus Beobachtungen von Coachingprozessen stammen und sich somit auf die Praxeologie beziehen.

Als erstes konstitutives Merkmal von Coaching wird gefordert, dass der Sprechanteil der Klientinnen höher ist als derjenige der Coachs. In den hier untersuchten Prozessen hören Coachs nur knapp 46\% der Dauer des Coachingprozesses den Klientinnen zu. Folglich ist der Sprechanteil 
der Klientinnen, entgegen der Forderung von Geißler, etwas kleiner als der Anteil der Coachs.

Weiter fordert Geißler, dass mindestens $20 \%$ der Sprechhandlungen Metakommunikation sein soll. Er bezieht sich dabei auf alle Sprechhandlungen von Coach und Klient/ in und definiert die Metakommunikation breiter als die Kategorie Metakommunikation im vorliegenden Kategoriensystem. Doch auch wenn als Vergleichsgröße der gesamte inhaltliche Fokus Coachingprozess (welcher die Metakommunikation beinhaltet) herangezogen wird, so sind nur durchschnittlich $11 \%$ der Interventionen der Metkommunikation nach Geißler zuzuordnen. Die immer wieder geforderte Transparenz und gemeinsame Abstimmung zum Vorgehen im Coachingprozess, d. h. die Metakommunikation, nimmt in der vorliegenden Stichprobe somit weniger Gesprächseinheiten in Anspruch, als dies von Geißler gefordert wird. Ein Blick auf die einzelnen Prozesse zeigt auch hier große Unterschiede. Der Anteil Metakommunikation (als inhaltlicher Fokus Coachingprozess) beträgt je nach Prozess weniger als ein Prozent bis hin zu 19\%. Damit liegt allerdings selbst der höchste Wert noch knapp unter dem benannten Mindestwert.

Von den Sprechhandlungen, die sich nicht auf die Metakommunikation beziehen, sollen mindestens $40 \%$ aller Sprechhandlungen Fragen oder spiegelnde Beiträge sein. Wird dies in der vorliegenden Studie in die Interventionsformen Fragen und Reflexionen übersetzt, machen diese insgesamt $55 \%$ aus, liegen also über dem geforderten Wert. Nur in einem Prozess wurden die geforderten $40 \%$ nicht erreicht.

Weiterhin sollen nach Geißler mindestens $60 \%$ der Sprechhandlungen den Klienten als Handlungssubjekt thematisieren. Wird dies nur mit dem inhaltlichen Fokus Erleben und Verhalten des Klienten verglichen, liegt der Wert mit $47 \%$ unter dem geforderten Wert. Nimmt man den inhaltlichen Fokus Körperwahrnehmung/körperlicher Ausdruck hinzu, in welchem ebenfalls die Klientin im Zentrum steht, erreichen die Prozesse durchschnittlich 55\% (zwischen 33 und 74\%), wobei nur vier der neun Prozesse die geforderten $60 \%$ erreichen.

Wie oben diskutiert, konzentrieren sich die Coachs der vorliegenden Studie entweder auf das Erleben und Verhalten des Klienten oder auf die Sachebene/das Klientensystem. Aufgrund Geißlers Forderung müsste der Fokus beim Erleben und Verhalten des Klienten liegen. Die vorliegende Untersuchung bestätigt dies zumindest tendenziell. Die von Geißler geforderten Mindestwerte werden jedoch nicht in allen Fällen erreicht. Der inhaltliche Fokus eines Coachings, d. h. die zentrale Frage, was in einem Coaching hauptsächlich thematisiert wird, kann damit nicht geklärt werden.

Eine Übereinstimmung der Ergebnisse mit Geißler findet sich dagegen bei dessen Forderung, dass maximal 10\% aller Sprechhandlungen Selbstoffenbarungen des Coachs beinhalten sollen. In der vorliegenden Untersuchung machen Ratschläge und Meinungsäußerungen des Coachs durchschnittlich knapp 6\% aus. Die 10\%-Marke wurde lediglich von einem Coach überschritten.

Ebenfalls werden die definierten maximal $10 \%$ vermittelndes Wissen mit durchschnittlich knapp 2\% der Kategorie Instruktion in den vorliegenden Daten deutlich unterschritten und folglich in keinem Prozess erreicht.

Insgesamt bleibt festzustellen, dass die von Geißler (2013) dargestellten konstitutiven Merkmale in den Coachingprozessen der vorliegenden Stichprobe nur teilweise bestätigt werden. Dies kann als Hinweis darauf gelten, dass ein unterschiedliches Coachingverständnis die Coachingpraxis prägen. Diese Hypothese wird zusätzlich durch die Heterogenität der Ergebnisse gespeist. Eine vertiefte Diskussion über den Coachingbegriff und ein unterschiedliches Verständnis von Coaching überschreitet allerdings den Rahmen des vorgetragenen Analyseschritts und soll nach der Auswertung der Daten der weiteren Stichprobengruppen sowie der Aktivitäten der Klientin vorgenommen werden, wie dies im anschließenden Ausblick dargestellt wird.

\section{Kritik und Ausblick}

Das Besondere der Stichprobe ist, dass es sich um CoachingExperten handelt, welche durch ihre Präsenz das Coachingverständnis im deutschsprachigen Raum prägen. Die Einbettung der Coachings in Ausbildungen oder Kongresse könnte Einfluss auf die Ergebnisse haben. Es ist denkbar, dass sich der Anteil an Metakommunikation reduziert, weil vorab der Ablauf und Hintergrund des Vorgehens besprochen wurde.

Im dem Artikel zu Grunde liegenden Promotionsprojekt werden drei Stichprobengruppen untersucht: 1) die hier dargestellte Gruppe der Coaching-Experten, 2) etablierte Coachs, mit Coachings aus ihrem ,Alltag' und 3) Masterstudierende der Angewandten Psychologie als Coachs, die im Rahmen ihres Studiums verschiedene aufeinander aufbauende Kurse zu den Themen Beratung und Coaching besuchten. Im letzten Kurs führten sie durch Supervision begleitete Coachings durch. Diese drei Stichprobengruppen ermöglichen eine ökologische Validierung auch an längeren Coachingprozessen. Zudem können mögliche Unterschiede im Einsatz der Interventionen von erfahrenen Coachs und Novizen (MSc-Studierenden) eruiert werden. Dies ist auch insofern relevant, als Prozessforschung oft auf Daten mit Studierenden in der Rolle des Coachs zurückgreift.

Mit Hilfe des KategorienSystem zur Analyse von Coachingprozessen - Perspektive Klientin (KaSyCo-K) (Deplazes 2015) werden zusätzlich die Aktivitäten der 
Klientin analysiert. Der Abgleich der Interventionen der Coachs mit den Aktivitäten der Klientin kann Hinweise zur Interaktion im Coachingprozess geben.

Längerfristig wäre die Zusammenarbeit mit anderen Coachingforschenden aufschlussreich, um die beiden $\mathrm{KaSyCo}$ mit weiteren Analysemöglichkeiten von Coachingprozessen zu kombinieren. Als weiterer Schritt soll eine Verbindung zur Wirksamkeitsforschung aufgebaut werden. Es ist denkbar, daraus best practice Vorgehen abzuleiten.

Open Access Dieser Artikel wird unter der Creative Commons Namensnennung 4.0 International Lizenz (http://creativecommons. org/licenses/by/4.0/deed.de) veröffentlicht, welche die uneingeschränkte Nutzung, Verbreitung und Wiedergabe für beliebige Zwecke erlaubt, sofern Sie den/die ursprünglichen Autor(en) und die Quelle ordnungsgemäß nennen, einen Link zur Creative Commons Lizenz beifügen und angeben, ob Änderungen vorgenommen wurden.

\section{Literatur}

Argyris, C. (1970). Intervention theory and method: A behavioral science view. Massachusetts: Addison-Wesley.

Backhausen, W., \& Thommen, J.-P. (2006). Coaching. Durch systemisches Denken zu innovativer Personalentwicklung (3. Aufl.). Wiesbaden: Gabler.

Behrendt, P. (2004). Wirkfaktoren im Psychodrama und Transfercoaching. Freiburg: Albert-Ludwigs-Universität.

Bortz, J., \& Döring, N. (2006). Forschungsmethoden und Evaluation: für Human- und Sozialwissenschaftler (4. Aufl.). Heidelberg: Springer.

Böning, U. (2014). Business-Coaching mit Führungskräften. In R. Wegener, M. Loebbert, \& A. Fritze (Hrsg.), Coaching-Praxisfelder. Forschung und Praxis im Dialog (S. 21-41). Wiesbaden: Springer.

De Meuse, K. P., Dai, G., \& Lee, R. J. (2009). Evaluating the effectiveness of executive coaching: Beyond ROI? Coaching, 2(2), 117-134. doi:10.1080/17521880902882413.

Deplazes, S. (2015). KaSyCo - Kategoriensysteme zur Analyse von Coachingprozessen. Instrument-Entwicklung und Anwendungsbeispiele. Disseration Universität Kassel.

Fillery-Travis, A., \& Lane, D. (2006). Does coaching work or are we asking the wrong question? International Coaching Psychology Review, 1(1), 24-36.

Geißler, H. (2009). Die inhaltsanalytische „Vermessung“ von Coachingprozessen. In B. Birgmeier (Hrsg.), Coachingwissen. Denn sie wissen nicht was sie tun? (S. 93-125). Wiesbaden: VS Verlag für Sozialwissenschaften.

Geißler, H. (2013). Produkt-und Marktsegmentierung von Coaching. In R. Wegener, A. Fritze, \& M. Loebbert (Hrsg.), CoachingPraxisfelder. Forschung und Praxis im Dialog - Online-Teil (S. 260-271). Wiesbaden: Springer. Retrieved from http://www. springer.com.

Gessnitzer, S., \& Kauffeld, S. (2014). Gefühle und Selbstoffenbarungen: Erfolgsfaktoren im Coaching (pp. 1-29). Presented at the 48. Kongress der Deutschen Gesellschaft für Psychologie, 23.27.09.2012, Bielefeld.

Graf, E. (2015). The discourses of executive coaching. Linguistic insights into emotionally intelligent coaching. Klagenfurt: Habilitationsschrift Alpen-Adria Universität.

Graf, E.-M., Aksu, Y., \& Rettinger, S. (2010). Qualitativ-diskursanalytische Erforschung von Coaching-Gesprächen. Organisationsberatung, Supervision, Coaching, 17, 133-149. doi:10.1007/ s11613-010-0188-7.
Grawe, K., Donati, R., \& Bernauer, F. (1994). Psychotherapie im Wandel. Von der Konfession zur Profession. Göttingen: Hogrefe.

Greif, S. (2008a). Coaching und ergebnisorientierte Selbstreflexion. Theorie, Forschung und Praxis des Einzel- und Gruppencoachings. Göttingen: Hogrefe.

Greif, S. (2008b). Die härtesten Forschungsergebnisse zum Coachingerfolg kennen und praktisch nutzen! Coaching-Magazin, 3, 46-49.

Greif, S. (2009). Grundlagentheorien und praktische Beobachtungen zum Coachingprozess. In B. Birgmeier (Hrsg.), Coachingwissen. Denn sie wissen nicht was sie tun? (S. 129-144). Wiesbaden: VS Verlag für Sozialwissenschaften.

Greif, S., Schmidt, F., \& Thamm, A. (2010). The Rating of Eight Coaching Success Factors - Oberservation Manual. Retrieved from http://www.home.uni-osnabrueck.de/sgreif/ veroeffentlichungen.html.

Greif, S., Schmidt, F., \& Thamm, A. (2012). Warum und wodurch Coaching wirkt. Organisationsberatung, 19(4), 375-390. doi:10.1007/s11613-012-0299-4.

Ianiro, P., \& Kauffeld, S. (2011). Black-Box Coaching-Prozess: Beziehungsaufbau und -gestaltung auf Grundlage interpersonaler Basisdimensionen. Presented at the LOCCS-Symposium, LMU München.

Ianiro, P. M., Schermuly, C. C., \& Kauffeld, S. (2013). Why interpersonal dominance and affiliation matter: An interaction analysis of the coach-client relationship. Coaching, 6(1), 25-46. doi:10.10 80/17521882.2012.740489.

Joo, B.-K. B. (2005). Executive coaching: A conceptual framework from an integrative review of practice and research. Human Resource Development Review, 4(4), 462-488.

Künzli, H. (2009). Wirksamkeitsforschung im FührungskräfteCoaching. Organisationsberatung, Supervision, Coaching, 16(1), $1-15$.

Mayring, P. (2003). Qualitative Inhaltsanalyse. Grundlagen und Techniken (8. Aufl.). Weinheim: Beltz.

Mayring, P. (2008). Neuere Entwicklungen in der qualitativen Forschung und der Qualitativen Inhaltsanalyse. In P. Mayring \& M. Gläser-Zikuda (Hrsg.), Die Praxis der Qualitativen Inhaltsanalyse (2. Aufl., S. 7-18). Weinheim: Belz.

Möller, H. (2001). Was ist gute Supervision? Grundlagen - Merkmale - Methoden. Stuttgart: Klett-Cotta.

Möller, H., \& Kotte, S. (2011). Die Zukunft der Coachingforschung. Organisationsberatung, Supervision, Coaching, 18(4), 445-456. doi:10.1007/s11613-011-0256-7.

Neumann-Wirsig, H. (2009). Supervisions-tools. Bonn: ManagerSeminare.

Radatz, S. (2003). Beratung ohne Ratschlag. Wien: Verlag Systemisches Management.

Rauen, C. (2009). Coaching-Tools II. Erfolgreiche Coaches präsentieren Interventionstechniken aus ihrer Coaching-Praxis (2. Aufl.). Bonn: ManagerSeminare.

Rauen, C. (2011). Coaching-Tools. Erfolgreiche Coaches präsentieren 60 Interventionstechniken ihrer Coaching-Praxis (11. Aufl.). Bonn: ManagerSeminare.

Riedel, J. (2003). Coaching für Führungskräfte: Erklärungsmodell und Fallstudien. Wiesbaden: Deutscher Universitätsverlag.

Schreyögg, A. (2009). Die Wissensstruktur von Coaching. In B. Birgmeier (Hrsg.), Coachingwissen. Denn sie wissen nicht was sie tun? (S. 47-60). Wiesbaden: Verlag für Sozialwissenschaften.

Schreyögg, A. (2010). Ist Coaching reine Prozessberatung oder sind hier auch andere Beratungsmodelle relevant? Organisationsberatung, Supervision, Coaching, 17(2), 119-132. doi:10.1007/ s11613-010-0185-x.

Schreyögg, A. (2012). Coaching. Eine Einführung für Praxis und Ausbildung (7. Aufl.). Frankfurt: Campus. 
Seiger, C., \& Künzli, H. (2013). Praxisfelder im Coaching: Beratung ohne Grenzen? In R. Wegener, A. Fritze, \& M. Loebbert (Hrsg.), Coaching-Praxisfelder. Forschung und Praxis im Dialog (S. 283294). Wiesbaden: Springer.

Stein, I. F. (2009). Which hat am I wearing now? An evidence-based tool for coaching self-reflection. Coaching: an International Journal of Theory, Research and Practice, 2(2), 163-175. doi:10.1080/17521880903102233.

Storch, M. \& Krause, F. (2002). Selbstmanagement - ressourcenorientiert. Grundlagen und Trainingsmanual für die Arbeit mit dem Zürcher Ressourcen Modell. Huber: Bern.

Strikker, F., \& Flore, M. B. (2010). Systematisierung von CoachingTools. In C. Heidsiek \& J. Peterson (Hrsg.), Organisationslernen im 21. Jahrhundert. Festschrift für Harald Geißler (S. 139-151). Frankfurt a. M.: Peter Lang.
Theeboom, T., Beersma, B., \& van Vianen, A. E. M. (2014). Does coaching work? A meta-analysis on the effects of coaching on individual level outcomes in an organizational context. The Journal of Positive Psychology, 9(1), 1-18. doi:10.1080/174397 60.2013.837499.

Wechsler, T. (2012). Das coachingtool-spezifische Wirkfaktorenmodell. Organisationsberatung, Supervision, Coaching, 19(4), 405-423. doi:10.1007/s11613-012-0305-x.

Willke, H. (1999). Systemtheorie II: Interventionstheorie (3. Aufl.). Stuttgart: Lucius \& Lucius. 